\title{
Essais
}

Revue interdisciplinaire d'Humanités

$15 \mid 2019$

Jouer l'histoire

\section{Représenter et transmettre la Première Guerre mondiale dans les jeux vidéo}

Retranscrire un conflit à la mémoire encore vive et diverse dans Soldats Inconnus : Mémoires de la Grande Guerre et Verdun 1914-1918

\section{Antoine Maillard}

\section{OpenEdition}

\section{Journals}

Édition électronique

URL : http://journals.openedition.org/essais/1593

DOI : $10.4000 /$ essais. 1593

ISSN : 2276-0970

Éditeur

École doctorale Montaigne Humanités

Édition imprimée

Date de publication : 15 octobre 2019

Pagination : 93-106

ISBN : 979-10-97024-07-9

ISSN : 2417-4211

Référence électronique

Antoine Maillard, «Représenter et transmettre la Première Guerre mondiale dans les jeux vidéo », Essais [En ligne], 15 | 2019, mis en ligne le 05 octobre 2020, consulté le 12 octobre 2020. URL : http:// journals.openedition.org/essais/1593; DOl : https://doi.org/10.4000/essais.1593 


\section{Représenter et transmettre la Première Guerre mondiale dans les jeux vidéo \\ Retranscrire un conflit à la mémoire encore vive et diverse dans Soldats Inconnus : Mémoires de la Grande Guerre et Verdun 1914-1918}

Antoine Maillard

La Première Guerre mondiale est un conflit qui a été pendant longtemps peu représenté dans les jeux vidéo. Elle a été souvent éludée par d'autres conflits comme la Seconde Guerre mondiale, la Guerre Froide, ou encore la guerre de Sécession qui représentent une grande proportion des productions vidéoludiques (Figure 1). Cependant, depuis le début des commémorations du Centenaire de la Grande Guerre, cet écart tend à se réduire. Bien sûr sa part de représentation est encore limitée et reste bien éloignée de celle de la Seconde Guerre mondiale (Figure 1). Le peu de jeux vidéo autour de ce conflit peut s'expliquer par des difficultés de représentation de cet événement, éléments qui ont été étudiés par les historiens Julien Lalu ${ }^{1}$, Chris Kempshall ${ }^{2}$, Thomas Rabinot ${ }^{3}$ ou encore Adam Chapman ${ }^{4}$.

Ces difficultés peuvent se résumer en trois grandes catégories : tout d'abord la spécificité du conflit qui reste dans l'imaginaire collectif comme une guerre de positions majoritairement composée d'attente, bien qu'ayant connu des phases de guerre de mouvement. Cette spécificité peut faire craindre aux développeurs de produire un jeu qui ne serait pas assez dynamique, sans réelle progression (gains territoriaux, avancée dans le territoire de l'armée adverse...) qui ferait comprendre au joueur qu'il participe à la victoire, ce qui pourrait donc rapidement le lasser. Une seconde limite pourrait être la mémoire qui entoure le conflit, mémoire qui est encore aujourd'hui vive et protéiforme. La Grande

1 Julien Lalu, "Représenter la Première Guerre mondiale dans les jeux vidéo : entre absence et uchronie ", Mission Centenaire 14-18 : http://centenaire.org/fr/espace-scientifique/societe/ representer-la-premiere-guerre-mondiale-dans-les-jeux-video-entre.

2 Chris Kempshall, The First World War in Computer Games, Basingstoke, Hampshire, Palgrave Pivot, 2015.

3 Thomas Rabino, "Jeux vidéo et Histoire ", Le Débat, n 177-5, 20 novembre 2013, p. 110-116.

4 Adam Chapman, "It's Hard to Play in the Trenches: World War I, Collective Memory and Videogames ", Game Studies, 16-2, décembre 2016 : http://gamestudies.org/1602/articles/ chapman. 
Guerre reste vue dans de nombreux pays comme meurtrière et sans but clair, a contrario de la Seconde Guerre mondiale qui a pour but la défaite de l'Allemagne nazie. La Première Guerre mondiale devient dès lors entourée d'une aura quasi sacrée qui pourrait faire peur aux développeurs de médias ayant pour objectif le divertissement. Enfin, une dernière limite serait la vision du soldat de ce conflit présent dans l'imaginaire collectif : des hommes qui ne sont pas maîtres de leur destin, dont leur habilité au combat ne permettra pas d'influencer leur survie et qui se feront invariablement tuer. Pendant très longtemps, les jeux autour de la Première Guerre mondiale sont restés restreints à des jeux de simulations aériennes comme Red Baron II (Dynamix, États-Unis, 1997) ou des jeux de stratégie comme History Line 1914-1918 (Blue Byte Software, Allemagne, 1992). Cela permettait aux développeurs de s'affranchir des difficultés de représentation de ce conflit en éloignant le joueur des tranchées.

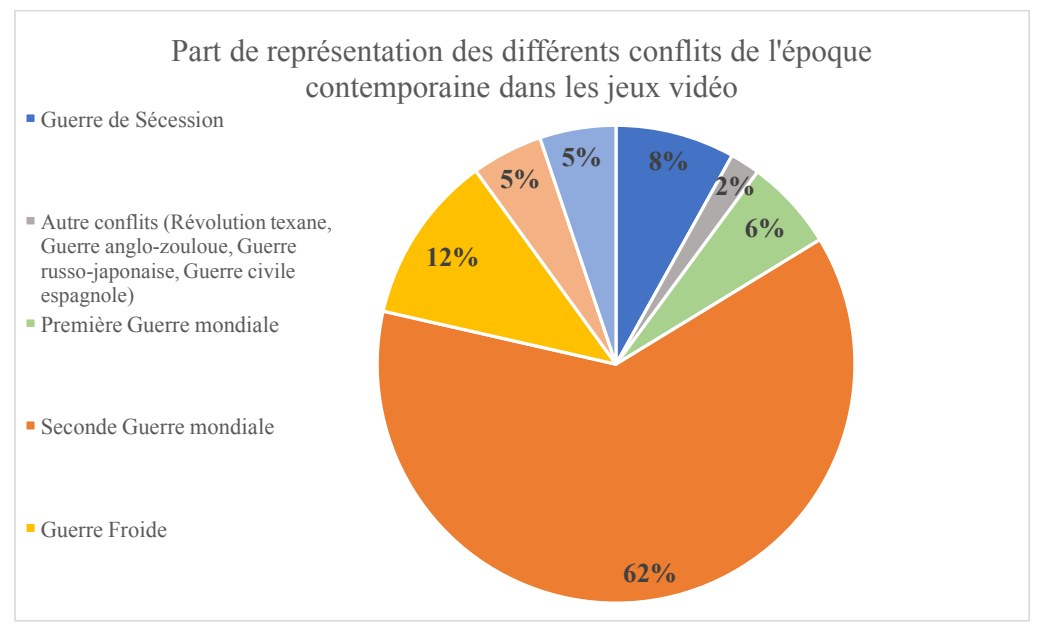

Figure 1 : Graphique réalisé grâce au recensement des jeux vidéo représentant la période contemporaine de l'histoire effectué par le site internet HistorioGames : http://www.histogames.com/ HTML/inventaire/periodes-historiques/epoque-contemporaine.php (consulté le 20/10/18)

Malgré ces limites, le début du Centenaire de la Première Guerre mondiale a vu apparaître de plus en plus de jeux vidéo centrés sur ce conflit majeur $\mathrm{du} \mathrm{XX}^{\mathrm{e}}$ siècle. Les commémorations ont remis sur le devant de la scène cet évènement historique incitant les développeurs à produire de nouveaux concepts. On retrouve ainsi des jeux comme Battlefield 1 (DICE, Suède, 2016), Battle of Empire 1914-1918 (Great War Team, Ukraine, 2015) ou encore 11-11 Memories retold (Digixart, France, 2018). Retranscrire la Grande Guerre impose aux développeurs d'employer de nombreuses ressources afin de recréer au mieux son environnement pour le joueur. Au fil de leurs lectures, de leurs découvertes, de leurs recherches, mais aussi de leur ressenti, ils vont faire des choix qui orientent leur représentation de la Grande Guerre. Trois inter- 
rogations apparaissent donc après ce constat. Tout d'abord, quels sont les différentes supports (sources, documentaires, films, bande-dessinées...) que les développeurs utilisent et qui les influencent lors de la conception d'un jeu. Deuxièmement, de quelle manière, en exploitant leurs recherches, ils représentent ce conflit dans leurs productions vidéoludiques. Le terme représenter doit se définir ici comme l'action de faire " figurer quelque chose par un moyen artistique ou autre procédé ${ }^{5}$. Enfin quel est le discours qu'ils adoptent sur la mémoire de la Grande Guerre.

Nous allons nous centrer dans cet article sur deux jeux vidéo qui m’ont particulièrement marqué et qui racontent la Grande Guerre de manière totalement différente. Tout d'abord, nous allons étudier Soldats Inconnus : Mémoires de la Grande Guerre (Soldats Inconnus) développé par Ubisoft Montpellier en 2014 avec le soutien de la Mission du Centenaire. Le joueur incarne ici quatre personnages : un Français, une Belge, un Américain et un Allemand dans de nombreux niveaux représentant les grandes batailles et évènements de la guerre sur le Front Ouest. Ce jeu vidéo d'action aventure, aux graphismes proches de la bande dessinée, adopte une spécificité qui a fait parlé de lui dans la presse, outre le fait qu'il soit une production française : le centre du gameplay n'est pas la mise à mort des combattants ennemis. Puis, le jeu de tir en vue subjective Verdun 1914-1918 (Verdun), paru en 2015 et développé par Blackmill Games et M2H, studios basés aux Pays-Bas depuis 2013. Il est décrit comme "le premier FPS [Jeu de tir en vue subjective] multijoueur se déroulant dans un environnement réaliste au sein de la Première Guerre mondiale " ${ }^{6}$. Ce travail est issu d'un mémoire réalisé sous la direction de Caroline Moine en 2017 à l'université de Versailles-Saint-Quentin-en-Yvelines, intitulé Les représentations de la Première Guerre mondiale dans les jeux vidéo : entre enjeux mémoriels et divertissement, qui se base sur un corpus de sources plus important, notamment des entretiens avec les développeurs des jeux Soldats Inconnus et Verdun. Nous étudierons dans un premier temps l'angle qu'adopte les développeurs de ces deux jeux vidéo pour immerger le joueur dans la Première Guerre mondiale, puis nous prendrons l'exemple de Verdun pour étudier la représentation vidéoludique de la Grande Guerre. Nous terminerons cet article par l'analyse de la place de la mémoire dans ces productions vidéoludiques.

5 Définition du verbe "représenter » sur le dictionnaire Larousse en ligne : https://www.larousse. fr/dictionnaires/francais/repr\%C3\%A9senter/68486?q=repr\%C3\%A9senter\#67737.

6 Page de vente du jeu Verdun sur la plateforme dématérialisée Steam http://store.steampowered. com/app/242860/?1=french (consulté le 08/10/2018). 


\section{Verdun 1914-1918 et Soldats Inconnus : mémoires de la Grande Guerre : deux choix différents de représentation de la Première Guerre mondiale}

Les jeux Verdun et Soldats Inconnus sont l'illustration des partis pris adoptés par les développeurs pour représenter la Première Guerre mondiale, avec chacun leur vision différente du conflit. Le jeu d'Ubisoft Montpellier, sorti en 2014, adopte une vision que l'on pourrait qualifier de pacifiste, avec une volonté de montrer aussi bien les soldats de première ligne que l'ensemble des acteurs du conflit. Cela se retrouve dans le fait que le joueur incarne aussi bien un soldat français qu'un soldat allemand, un volontaire américain, ou encore une infirmière belge. Le joueur traverse de nombreux espaces tout au long de son aventure, qu'il s'agisse des tranchés, mais aussi des villes et des zones occupées. De nombreuses thématiques sont abordées, comme la mobilisation, le sort des prisonniers, les attaques de gaz, etc. Ce jeu se présente donc sous la forme d'un large tableau qui représente le conflit. Le parti pris pacifiste se retrouve largement dans un élément du jeu qui a suscité l'engouement à son encontre : les développeurs ont essayé d'éviter de centrer le gameplay sur la mise à mort, choix qui peut paraître paradoxal pour la représentation d'un conflit dans laquelle le joueur incarne un soldat de première ligne. De nombreux journalistes se sont intéressés à cette production, louant sa manière de représenter la Grande Guerre, bien éloignée des traditionnels jeux de tirs ${ }^{7}$. Pour les développeurs, ce choix permettait de ne pas banaliser la mort dans le jeu et ainsi rendre celle des personnages plus marquante. On retrouve cette idée lors de la bataille du Chemin des Dames, qui clôt l'histoire et aboutit au sort du personnage principal du jeu, Émile, condamné à mort après avoir tué l'officier qui l'obligeait à suivre un ordre qu'il jugeait absurde.

Paul Tumelaire, directeur artistique de Soldats Inconnus, a contribué au choix de ce propos, car il a été influencé par divers médias qu'il a consultés pour préparer le développement du jeu. On retrouve tout d'abord dans ses influences la bande dessinée de Manu Larcenet, Une aventure rocambolesque de Vincent Van Gogh : La ligne de Front, parue en 2004. Cette bande dessinée adopte un ton ouvertement pacifiste par le biais d'une histoire imaginaire se déroulant pendant la Première Guerre mondiale. Ce travail a beaucoup inspiré les développeurs du jeu qui ont choisi d'adopter les codes de la bande dessinée pour créer les graphismes de Soldats Inconnus. Ils se sont notamment inspirés de la réflexion de Manu Larcenet sur les couleurs. Dans la bande dessinée, les couleurs sont vives lorsque les personnages sont loin du front et s'assombrissent lorsqu'il s'en rapproche. Cette inspiration se retrouve lors

7 Cyril Bonnet, "Soldats Inconnus : un plaidoyer vidéoludique contre la guerre ", Nouvelobs, 2014 : https://teleobs.nouvelobs.com/jeux-video/20140627.OBS2020/soldats-inconnus-unplaidoyer-videoludique-contre-la-guerre.html (consulté le 21/10/2018). 
du troisième niveau dans lequel le joueur, par l'intermédiaire d'Émile, se retrouve confronté pour la première fois à la guerre. On observe cette prise de conscience dans le changement des couleurs tout au long du niveau qui marque la réelle entrée du personnage dans le conflit. La seconde influence des développeurs est l'émission de Daniel Mermet diffusée sur France Inter jusqu'en 2014 : Là-Bas si jóy suis. Son présentateur y aborde abondamment des sujets tels que les fusillés ou le rôle des industriels dans le déclenchement de la guerre, ces médias adoptant eux même une vision pacifiste sur ce conflit. Les développeurs expliquent aussi ce choix par une volonté de rompre avec ce qui se faisait habituellement dans les jeux vidéo, en banalisant la mort au travers des jeux de tirs. Les développeurs affirment ne pas avoir voulu faire un jeu de guerre, mais réaliser un jeu sur la guerre, ce qui est parfois problématique puisque certaines séquences du jeu consistent à participer aux combats.

Verdun adopte quant à lui une vision à l'exact opposé, car les combats sont ici au centre du jeu. Il a été développé principalement par une petite équipe originaire des Pays-Bas qui a fait le choix de réaliser un jeu sur la Grande Guerre, car les développeurs avaient pour habitude de se rendre dans la région de Verdun et étaient impressionnés par les stigmates du conflit, toujours présents. Le choix du titre Verdun avait pour eux une certaine symbolique : dans leur esprit, ce nom est associé à l'exemple même de la bataille représentant l'horreur de cette guerre, en Allemagne et en France, mais aussi dans la mémoire européenne. Verdun se veut réaliste dans son gameplay; les développeurs ont fait le choix de réaliser un jeu de tir en vue subjective : ce gameplay était pour eux le meilleur moyen d'immerger complètement le joueur dans cette période historique. La difficulté est omniprésente, car une seule balle peut tuer le joueur, ce dernier devant faire preuve de prudence pour atteindre ses objectifs. Quant aux parties, celles-ci se déroulent à la manière d'un match : sur un terrain défini et limité, les équipes doivent chacune à leur tour essayer de capturer les tranchées adverses, la partie se terminant à la fin du temps imparti ou lorsque toutes les tranchées ont été capturées par l'une des deux équipes. Ce jeu vidéo est vu comme la référence en termes de réalisme sur les combats d'infanterie de la Première Guerre mondiale. Cependant, il s'agit d'un jeu dit de "niche ", c'est-à-dire qui cible un public très spécifique en raison de sa difficulté, de son gameplay et de sa volonté de représenter fidèlement la Première Guerre mondiale.

\section{L'exemple de Verdun, la mémoire par le matériel et les environ- nements}

Les développeurs de Blackmill Games et de $\mathrm{M} 2 \mathrm{H}$ ont fait le choix de créer un jeu vidéo qui cherche à retranscrire de manière fidèle la Première Guerre mondiale et ont de fait reproduit les champs de batailles du Nord de 
la France. L'aire de jeu dans laquelle le joueur évolue est très importante pour son immersion, car dans le cas de la Première Guerre mondiale le terrain n'est pas juste un élément décoratif. Comme le relève Chris Kempshall :

"Le terrain qui accueille la Première Guerre mondiale n'est pas juste un cadre ou une scène où le conflit se déroule. Il est un participant actif de la guerre et un transmetteur de mémoire. La boue écœurante, les trous d'obus, les tranchées ruinées, les champs et les villes, ce ne sont pas des éléments passifs de la guerre ; ils sont actifs et dangereux. $\|^{8}$

Pour reproduire ces espaces, les développeurs ont dû se documenter. Ils se sont dans un premier temps rendus sur place pour parcourir les champs de bataille, par exemple le fort de Douaumont qui est aujourd'hui visitable et qui est le centre d'une aire de jeu dans Verdun. Puis, à l'aide d'archives (photographies, plans, peintures...) et de documents qu'ils ont créés lors de leur visite, ils ont réalisé des croquis qui permettent de reconstituer en 3D les champs de bataille (Figure 2) ${ }^{9}$. Ces croquis se présentent sous la forme d'une carte représentant l'aire de jeu en entier, chaque élément de cette carte (tranchée, aspérité...) est documenté par une archive. Chacune des sources utilisées permet de constituer une partie précise de l'aire de jeu dans laquelle les joueurs évolueront ensuite (Figure 3). Le résultat est la création de champs de batailles avec de nombreuses tranchées, barbelés, etc. Le terrain est difficile à traverser sous le feu ennemi et les tranchées sont séparées par un no man's land parsemé de trous d'obus. La morphologie de l'aire de jeu devient donc ici un élément essentiel, car elle peut tout aussi bien être fatale lorsque le joueur se coince dans les barbelés, ou lui permettre de se protéger des tirs ennemis lorsqu'il se cache dans un trou d'obus. Il était important, pour les développeurs, de varier les aires de jeux (La Marne, les Vosges, le Fort de Douaumont...) et d'éviter une représentation unique et stéréotypée des champs de batailles de la Grande Guerre qui seraient recouverts par la boue et labourés par les obus. Cette pluralité permet d'éviter une lassitude des joueurs par le changement constant d'espace de jeu et la variété des environnements (Figure 4). Il était très difficile de réaliser cet aspect dans Verdun qui se concentre uniquement sur les combats du Nord de la France, là ou d'autre jeux comme Battlefield 1 ont choisi de représenter des espaces variés allant du Nord de la France jusqu’à l'Empire Ottoman. Les développeurs de Verdun revendiquent aujourd'hui la représentation de plus $500000 \mathrm{~m}^{2}$ de champs de bataille ${ }^{10}$.

8 Chris Kempshall, The First World War in Computer Games, op. cit., p. 50-51.

9 L'ensemble de ces croquis sont disponibles dans, Charlie Hall, «In the trenches: Behind the scenes of Verdun, and a preview of the next expansion ", Polygon, 16 septembre 2015 : https:// www.polygon.com/features/2015/9/16/9326279/verdun-behind-the-scenes-preview-horrorsof-war (consulté le 21/10/2018).

10 Information présente dans les temps de chargement du jeu Verdun. 


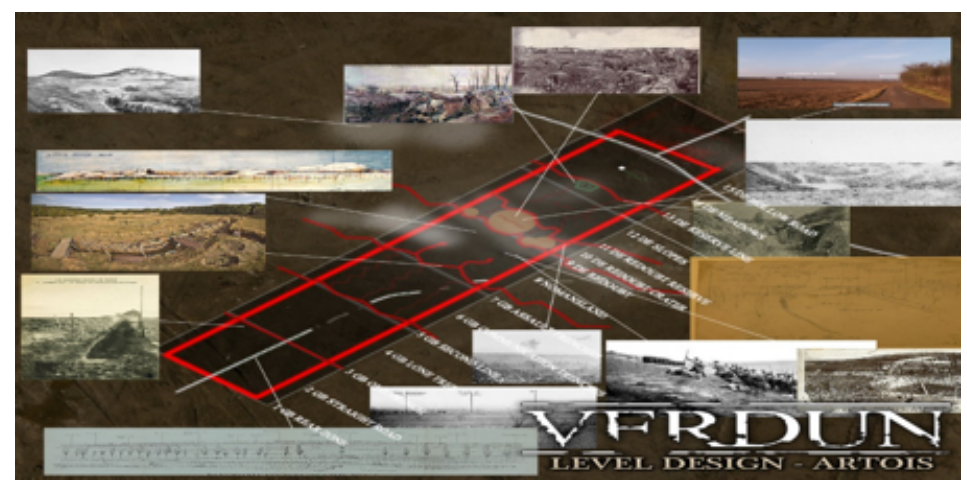

Figure 2 : Schéma produit par l'équipe de développement de Verdun afin de réaliser le level design de la carte Artois. Crédit : $M 2 H$ and Blackmill Games

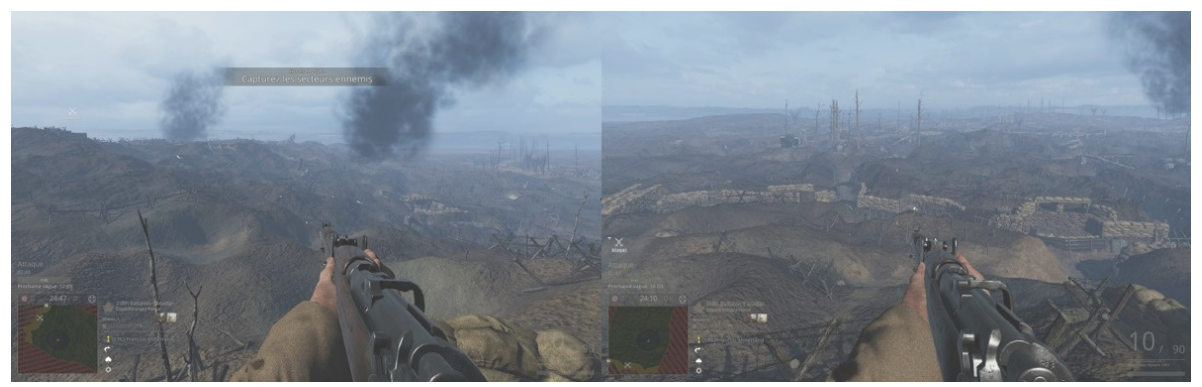

Figure 3 : Captures d'écran de l'aire de jeu Artois

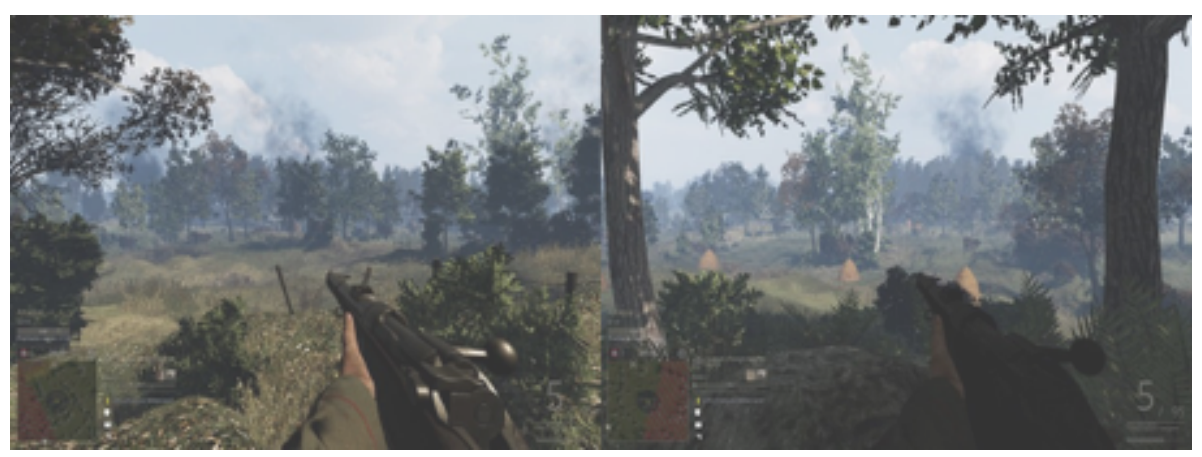

Figure 4 : Captures d'écran de l'aire de jeu Aisne

Outre la représentation des champs de batailles, celles des armes, des uniformes et du matériel des soldats participent aussi à l'immersion du joueur dans ce jeu vidéo. Tous ces éléments deviennent des symboles d'authenticité que le joueur pourra reconnaître et qui lui permettront de se sentir totalement immergé dans cette période. On peut dès lors retrouver des degrés de lecture différents du jeu en fonction des connaissances historiques du joueur. On y retrouve la plupart des unités de la Première Guerre mondiale présentes sur le front Ouest, tandis que du côté de l'Entente, sont présents les soldats belges, 
les soldats français ou encore, depuis peu, les forces de l'ANZAC ${ }^{11}$. Du côté de la Triple Alliance, la représentation se limite à des unités de l'armée allemande comme les Landser (Infanterie), les Alpenjäger (Chasseurs-alpins) ou encore les Pioniere (soldats du génie) (Figure 5).

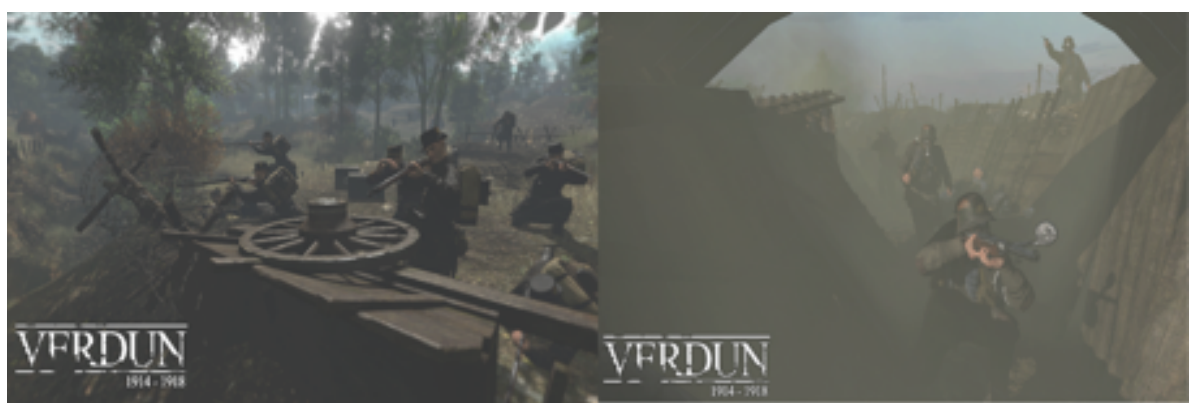

Figure 5 : Captures d'écran des troupes belges et allemandes Crédit : $M 2 H$ and Blackmill Games

Les uniformes et le matériel qui équipent les différentes unités sur la période de 1914 à 1918 ont été reproduits à l'aide de différents supports afin qu'ils demeurent au plus près de la réalité : les développeurs se sont appuyés sur les ouvrages de la collection Men-at-Arms de la maison d'édition Osprey et qui représentent tous les uniformes des différents belligérants ${ }^{12}$. En plus de ce support, les développeurs ont aussi fait appel à des associations de reconstitutions qui recréent les uniformes et le matériel que portaient les soldats de la Grande Guerre. Ce recours aux groupes de reconstitution se retrouve d'ailleurs chez de nombreux développeurs comme DICE pour Battlefield 1. Cette fidèle représentation fonctionne à la manière de points de repère qui permettent au joueur de se sentir immergé dans cette période historique. Il en est de même pour la modélisation des armes (Figure 6) qui sont l'un des points névralgiques $\mathrm{du}$ jeu, car elles touchent directement au gameplay et in fine au divertissement des joueurs, éléments qui conditionnent très souvent leur achat.

Les armes individuelles en dotation sont pour la plupart des fusils à verrou ou à répétition, éléments qui influent sur le gameplay ${ }^{13}$. Ce système impose un délai entre deux tirs, le joueur doit donc veiller à bien aligner sa cible sous peine de se voir exposé aux répliques de l'ennemi. Cela rend le jeu difficile à appréhender, notamment dans les combats à courte distance, car il impose de cibler et de tirer le premier pour abattre son ennemi. La représentation réaliste de ces

11 Australian and New-Zealand Army Corps.

12 Lien vers la page de vente des éditions Osprey pour la collection Man At Arms consacrée à la Première Guerre mondiale : https://ospreypublishing.com/store/military-history/series-books/ men-at-arms? filterPeriod=World\%20War\%201 (consulté le 08/10/2018).

13 Un fusil à verrou est un fusil où il est nécessaire d'ouvrir la culasse après le tir puis de la refermer, ce qui permet d'éjecter la douille de la balle précédente et d'y introduire la balle suivante pour effectuer un nouveau tir. 
armes, notamment à verrou, peut être vue comme nuisant au dynamisme du jeu, frustrante et donc rapidement lassante pour le joueur. D'autres productions comme Battlefield 1 se sont affranchis de cette limite en ne présentant que des armes automatiques et semi-automatiques individuelles pourtant bien moins présentes au cours de la Première Guerre mondiale, afin de garder une certaine nervosité. Pour l'historien Chris Kempshall, l'utilisation et la fidèle représentation de ces armes historiques est un élément qui est souhaité par l'audience ciblée. Cette représentation permet de créer un double sentiment d'authenticité et de compétence, car toucher un soldat ennemi en utilisant une arme authentique suggère au joueur qu'il aurait pu faire de même en temps de guerre ${ }^{14}$. Le public visé par ce jeu, un public de niche, dispose d'une certaine connaissance sur ce conflit, notamment des armements. Il prend ainsi plaisir à le retrouver et à l'utiliser. Les développeurs doivent donc, pour répondre à cette attente, reproduire ces armements le plus fidèlement possible et utilisent pour se faire différentes sources. La principale est la chaîne YouTube $C \&$ Rsenal ${ }^{15}$ qui documente et décrit les armes militaires historiques. Ces contenus YouTube, après avoir aidé les développeurs, tentent de s'adresser au public de ces jeux. Cela passe par la création de playlists et de vidéos spécialement dédiées aux armes présentes dans ces productions. C'est le cas de la chaîne CひoRsenal, qui propose une série dédiée aux armes de la Première Guerre mondiale et qui donne à ses abonnés la possibilité de rejoindre un serveur vocal dédié au jeu Verdun.

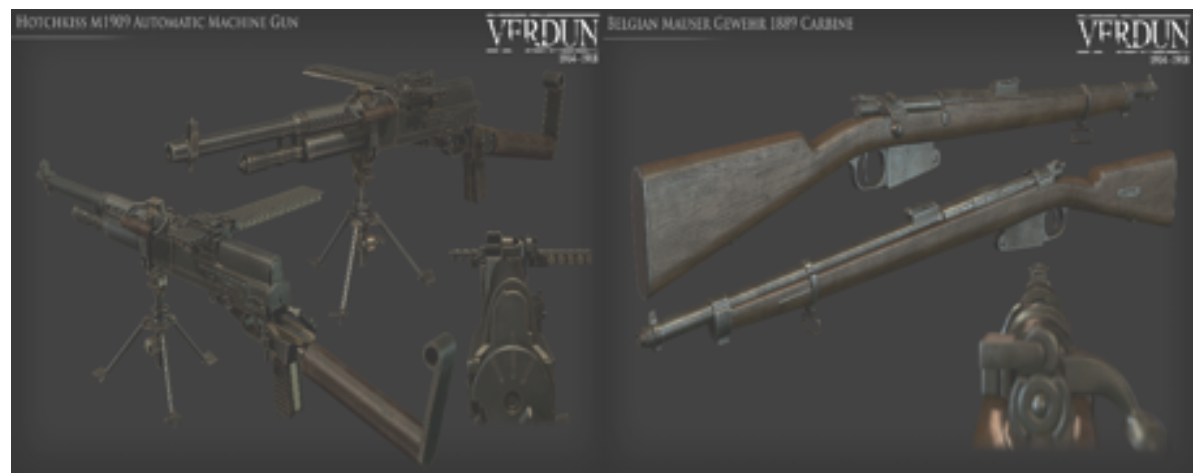

Figure 6 : Présentation des reproductions 3D de l'armement dans Verdun 1914-1918 Crédit : $M 2 H$ and Blackmill Games

14 Chris Kemshall, The First World War in Computer Games, op. cit., p. 72.

15 ChaineYouTube CerRsenal:https://www.youtube.com/channel/UClq1dvO44aNovUUy0SiSDOQ/ about (consulté le 08/10/2018). 


\section{La place de la mémoire de la Grande Guerre dans ces produits culturels mondialisés}

L'ensemble des jeux étudiés ici représentent le simple combattant, soldat de première ligne, confronté aux horreurs de la Première Guerre mondiale. Ils transcrivent un évènement historique encore largement ancré dans la mémoire collective. Cette mémoire se concentre avant tout sur des images très stéréotypées : on retrouve la tranchée présente sur le front Ouest et le discours a un intérêt plus particulier pour l'expérience personnelle des soldats, centrée notamment sur leurs souffrances. Cette mémoire de la Première Guerre mondiale est encore aujourd'hui sujette à de nombreuses controverses dans nos sociétés, notamment en France avec la question des fusillés. En Angleterre, toujours selon Chris Kempshall, la Grande Guerre est vue comme une mauvaise guerre, couplée à une image de guerre meurtrière, bien que $88 \%$ des soldats anglais en soient rentrés vivant ${ }^{16}$. En raison de l'importance de cette mémoire, produire un jeu vidéo sur cette période reste très difficile ; s'il est vu par le public comme étant insuffisamment respectueux de la Première Guerre mondiale, cela pourrait devenir une opération risquée pour un développeur, notamment en termes de ventes. Ceux qui ont souhaité réaliser un jeu vidéo sur ce conflit ont dû adapter leur production à cette spécificité mémorielle; chacun de ces jeux vidéo possède dès lors un message invitant au souvenir des combattants qui ont souffert au cours de ce conflit.

Dans Soldats Inconnus, cette mémoire est présente à de nombreuses reprises. Cependant, cette injonction au souvenir se retrouve principalement dans la conclusion du jeu et le discours qui y est tenu : "Même si leur corps est depuis longtemps retourné à la poussière, leur sacrifice continue de vivre en nous. Nous devons chérir leur mémoire, nous n'avons pas le droit d'oublier. » Ce moment marque la mort d'Émile, personnage auquel s'étaient attachés les joueurs. Il est accompagné d'une musique au piano et de la voix de Marc Cassot. Ce dernier n'est pas un doubleur inconnu en France; il est notamment connu par la communauté des joueurs cinéphiles adeptes, par exemple, du Seigneur des anneaux (Peter Jackson, 2001). Il est également la voix principale de plusieurs attractions du Puy du Fou, ce qui le lie davantage à l'histoire de la Première Guerre mondiale. Sa voix fait naître l'émotion chez le joueur, car il interprète souvent des personnages sages, un mentor ou encore un conteur. Il y a donc une réelle volonté, par l'utilisation de cet élément, de provoquer une réaction émotionnelle chez le joueur. Cela nous est confirmé par une vidéo montée par Yoan Fanise ${ }^{17}$, l'un des directeurs de l'équipe d'Ubisoft, qui rassemble la

16 Chris Kemshall, The First World War in Computer Games, op. cit., p 3.

17 Lien vers la vidéo Valiant Hearts Ending - Youtubers reactions [Spoiler] de Yoan Fanise : https:// www.youtube.com/watch?v=NeHFwBFi0Z8 (consulté le 08/10/2018). 
réaction de Youtubeurs sur la fin du jeu. On distingue donc réellement une sorte de morale, dispensée au joueur dans la conclusion qui se rapproche du "plus jamais ça ", ce qui fait entrer la narration dans le devoir de mémoire.

Dans Verdun, ce devoir de mémoire prend une tout autre forme : un événement communautaire est organisé chaque année pendant les fêtes de Noël. Intitulé Christmas Truce, cet événement fait référence à la trêve de Noël 1914. Une aire de jeu spéciale est dès lors ajoutée temporairement au jeu et rompt complètement avec le gameplay traditionnel de Verdun (Figure 7) : les joueurs n'y possèdent plus d'armes, mais des boules de neiges qu'ils peuvent se lancer ; un terrain de foot est présent au centre avec un ballon et de multiples stands permettent d'envoyer des cartes postales par mail à leurs proches. Le joueur y retrouve des scènes iconiques de représentation de cette trêve de Noël 1914 qu'il a pu voir, par exemple, dans le film de Christian Carillon, Joyeux Noël (Carillon, 2005, Figure 8). Ce gameplay est imposé au joueur, car il ne possède pas d'arme et le jeu l'empêche d'effectuer tout action hostile. Par cet événement communautaire, les joueurs sont invités à faire des dons à l'association War Child, qui s'occupe des enfants victimes de guerre. Les développeurs invitent donc les joueurs à agir sur le présent par le souvenir du passé en faisant des dons pour les conflits actuels. Cependant, la trêve ne dure pas, car une fois le temps imparti terminé, l'aire de jeu change et les joueurs doivent de nouveau s'entretuer. C'est là toute la différence entre jeu et réalité, car les équipes sont mobiles et après une trêve, les joueurs peuvent retourner s'affronter sans état d'âme. On retrouve ici l'une des principales limites des reconstitutions historiques ainsi que d'une représentation "réaliste » dans un jeu vidéo : l'absence de la peur de la mort. Mourir ici n'est pas une fin en soi, mais pousse le joueur à apprendre de ses erreurs et à recommencer pour mieux faire.

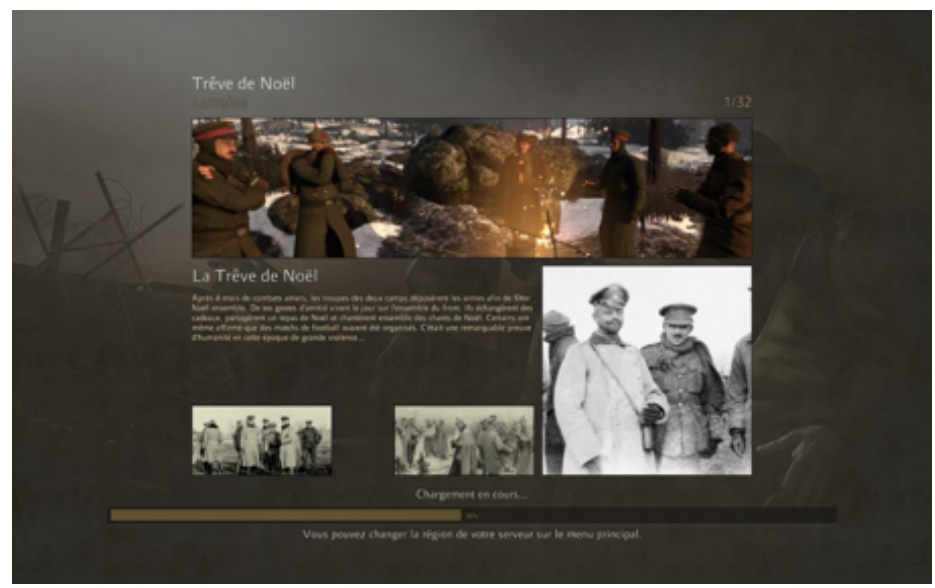

Figure 7 : Temps de chargement de l'aire de jeu Christmas Truce 


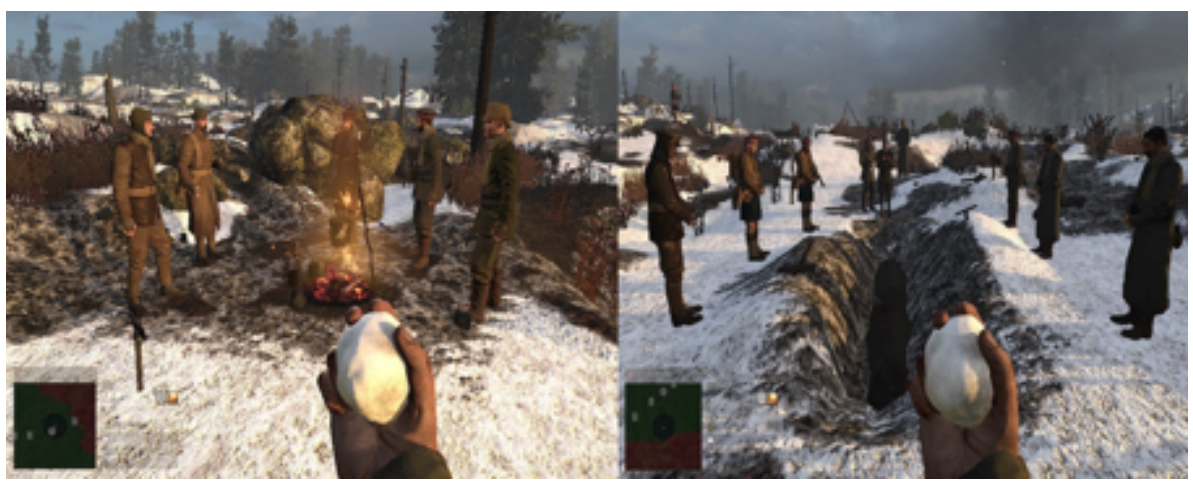

Figure 8 : Scènes de fraternisation de l'aire de jeu Christmas Truce

\section{Conclusion}

Verdun et Soldats Inconnus adoptent deux visions éloignées pour représenter la Première Guerre mondiale, l'un mettant au centre les combats et l'autre les souffrances que provoque la guerre. Ces deux jeux se rejoignent cependant sur un point : on y aperçoit une forme de "muséalisation " de l'histoire, concept développé par l'historien du cinéma Laurent Véray pour parler des films Un long dimanche de Fiançailles (Jeunet, 2004) et Joyeux Noël (Carion, $2005)^{18}$. Ces films mettent en avant un ensemble d'objets, d'anecdotes, d'idées qui ne permettent pas forcément de mieux comprendre le conflit, analyse qui semble totalement transposable à ces jeux vidéo. Ces représentations de l'histoire s'orienteraient donc plutôt vers l' histotainment ${ }^{19}$, néologisme anglosaxon utilisé par Nicolas Offenstadt pour parler des groupes de reconstitution. Il désigne l'histoire convoquée uniquement pour le divertissement, qui ne prend pas ou peu en compte l'explication du passé, la véritable transmission d'un savoir. Ce concept est le reflet de la transformation de l'histoire en produit culturel de masse qui n'épargne aucun domaine : bande-dessinées, télévision, cinéma, littérature, et jeu vidéo. Le divertissement par l'histoire passe ici, notamment dans Verdun, par la fidèle représentation des armes, des uniformes et des équipements, ainsi que par la représentation des champs de batailles qui sont des points de repère pour le joueur qui se retrouve transposé dans cette période. Mais cette authenticité dans les représentations du matériel et des équipements en dotation est une demande du public cible. Les développeurs de Verdun ont ainsi choisi d'inclure les troupes canadiennes en raison de l'importante communauté canadienne qui jouait à leur jeu.

18 Laurent Véray, La Grande Guerre au cinéma : de la gloire à la mémoire, Ramsay Cinéma, Paris, 2008, p. 212.

19 Ce terme est emprunté par Nicolas Offenstadt à l'historien allemand Wolfgang Hardtwig, auteur de Verlustder Geschichte - oder wie unterhaltsam ist die Vergangenheit?, Berlin, Vergangenheitsverlag, 2010. 
Concernant les sources et les influences qu'utilisent les développeurs, on s'aperçoit qu'ils se basent sur l'étude des journaux de marches, des photographies d'époques, des témoignages des combattants, des lettres, etc. Ils prennent donc la place des historiens en interprétant eux-mêmes les sources et en les utilisant pour créer de nouvelles représentations. On retrouve une certaine distance dans leur discours avec le monde de la recherche historique, qui est cependant à nuancer avec le recours aux conseils d'historiens par les studios de développement. Cette vision se retrouve aussi chez les développeurs de Battefield 1, pour qui l'histoire académique serait un univers plein de contraintes avec lequel il est plus difficile de collaborer qu'avec les " passionnés ${ }^{20}$. Soldats Inconnus et Verdun adoptent cependant un discours commun sur le conflit, invitant au souvenir des soldats morts. On observe une mise en avant d'individus (soldats et civils) jugés comme innocents. Ils sont les victimes d'un système inhumain qui les entraine dans une guerre atroce à laquelle ils sont obligés de participer et font preuve d'abnégation et se sacrifient pour leurs camarades. Cet élément fait qu'au-delà des aspects de divertissement, les développeurs tiennent cependant un vrai discours sur le conflit à la manière d'autres médias.

Antoine Maillard

Mission Histoire du département de la Meuse antoin.maillard@gmail.com

\begin{abstract}
Antoine Maillard a réalisé un mémoire intitulé Les Représentations de la Première Guerre mondiale dans les jeux vidéo : entre enjeux mémoriels et divertissement, sous la direction de Caroline Moine, en 2017, à l'université de Versailles-Saint-Quentin-en-Yvelines. Il est actuellement médiateur des collections et expositions, en service civique, à la Mission Histoire du département de la Meuse.
\end{abstract}

Thème de recherche : Représentation de l'Histoire dans les jeux vidéo

\title{
Résumé
}

La Première Guerre mondiale est un évènement qui a été largement sous-représenté dans les jeux vidéo face aux multiples productions qui s'intéressent à la Seconde Guerre mondiale. Cependant, depuis le début du Centenaire de la Grande Guerre, l'on voit une multitude de nouvelles productions autour de ce conflit. Par l'intermédiaire des jeux Soldats Inconnus : Mémoires de la Grande Guerre et Verdun 1914-1918, nous nous intéressons dans cet article au travail des développeurs pour représenter ce conflit majeur du XX⿳亠丷厂犬 siècle dans le média vidéoludique.

\section{Mots-clés}

Jeux vidéo, histoire des représentations, mémoire, Première Guerre mondiale, Histoire culturelle, Soldats Inconnus : Mémoires de la Grande Guerre, Verdun 1914-1918.

20 Stéphanie Trouillard, "Quand le jeu vidéo à succès Battlefield fait revivre la Grande Guerre ", France 24, 21 octobre 2016 : http://www.france24.com/fr/20161021-battlefield-1-jeu-videogame-premiere-guerre-mondiale-histoire-electronic-arts (consulté le 08/10/2018). 


\section{Abstract}

The First World War is an event that was largely underrepresented in video games compared to the multiple productions that are interested in the Second World War. However, since the beginning of the Centenary of the Great War, one can see a multitude of new productions around this conflict. Through Valiant Hearts: The Great War and Verdun 1914-1918, this article will focus on the work of developers that depicts this major conflict of the twentieth century in video games.

\section{Keywords}

Video games, History of representations, memory, First World war, cultural. History, Valiant Hearts: The Great War, Verdun 1914-1918. 